monográfico: Trasplante Renal. Experiencia del Hospital Clínico de Barcelona

Arch. Esp. Urol., 58, 6 (485-489), 2005

\title{
POTENCIALES BENEFICIOS DEL TRASPLANTE RENAL DE DONANTE VIVO
}

Juan M. Corral M olina, Pilar Luque Gálvez y Juan B. Alcover G arcía.

Servicio de Urología y Trasplante Renal. Hospital Clínico de Barcelona. Barcelona. España

Resumen.- El trasplante renal de donante vivo es la mejor opción terapeútica en la insuficiencia renal terminal, y aunque en nuestro país ha sido una opción infrautilizada, adquiere un papel importante en la reducción de las listas de espera para trasplante, puesto que la donación de cadáver es insuficiente. El trasplante renal de donante vivo ofrece múltiples ventajas respecto al de cadáver: mayor supervivencia del injerto y receptor a corto, medio y largo plazo, el hecho de ser un procedimiento programado permite optimizar las condiciones del donante y receptor, así como disminuir al mínimo el tiempo de isquemia entre la nefrectomía y el implante. Además, destaca la buena función inicial ( hasta un $90 \%$ ) sin necesidad de diálisis y la menor incidencia de rechazo, lo cual disminuye la necesidad de fármacos para prevenir el rechazo.
Palabras clave: Trasplante renal. Donante vivo. Evolución receptor. $N$ efrectomía laparoscópica.

Summary.- Living donor kidney transplantation is the best therapeutic option for endstage renal failure. In spite of being an underused option in our country, it acquires an important role reducing the waiting lists for transplantation because cadaver donation is not enough. Living donor kidney transplantation offers multiple advantages when compared with cadaver donor transplantation: longer graft and patient survival on the short, mid and long-term; the fact that a scheduled procedure allows us to optimize donor and receptor's conditions; and ischemia time between nephrectomy and transplantation can be shortened to a minimum. A good initial function without need of dialysis (up to $90 \%$ ) and lower incidence of rejection, which diminishes the need of antrejection drugs, should also be emphasized.

Keyw ords: Kidney transplantation. Living donor. Receptor 0 utcomes. Laparoscopic nephrectomy. 


\section{INTRODUCCIÓN}

El trasplante renal es actualmente considerado como la mejor opción en el tratamiento de la enfermedad renal en fase terminal (insuficiencia renal crónica, IRCT). Sin embargo, debido al progresivo aumento de pacientes que entran a formar parte de las listas de espera, el número de órganos disponibles a partir de donantes cadáver es insuficiente de cara a hacer frente a las necesidades de órganos para trasplante (1).

En 1997 en Estados Unidos por cada paciente trasplantado se añadían dos nuevos casos en lista de espera (2-7). Incluso en España, donde se muestra un aumento continuo en la tasa de donación (33.7 donantes por millón de población en 2002) hasta alcanzar el índice más alto en el mundo y que ha llegado a ser modelo de referencia, la alternativa de la donación de riñones de donante vivo se ofrece a pacientes seleccionados afectos de IRCT.

En España la obtención de órganos para trasplante renal a partir de donante vivo representa aproximadamente valores alrededor del $1 \%$ del total de donaciones, mientras que en otros países llega a representar más del $40 \%$ siendo la principal fuente de obtención de riñones para trasplante en A mérica Latina (2, 813). Teniendo en cuenta la persistente escasez de órganos para trasplantar, el progresivo aumento de buenos resultados obtenidos en el trasplante ( $T x$ ) de donante vivo renal, y la evolución de las pautas de inmunosupresión, los esfuerzos actuales deben encaminanarse a potenciar la obtención de riñones a partir de este tipo de donantes, sin olvidar las donaciones de cadáver.

Inicialmente la única opción terapeútica definitiva de los pacientes afectos de IRCT fue el Tx de donante vivo realizado mayoritariamente con riñones procedentes de hermanos HLA idénticos o bien gemelos univitelinos, debido a su excelente evolución aún en ausencia de inmunosupresión (14). Sin embargo, la experiencia acumulada y el descubrimiento de fármacos inmunosupresores amplió el rango de donación de vivo a familiares con menor compatibilidad, padres y hermanos no idénticos, con lo que se consiguió generalizar este tipo de $\mathrm{Tx}$, hasta el punto de que pueden ser llevados a cabo en sujetos no emparejados genéticamente (15). Una de las razones de mayor peso que en la actualidad justifica la práctica del Tx de donante vivo es por tanto el insuficiente número de donantes cadáver en relación con la demanda de Tx, por más que las razones de éste número insuficiente puedan ser diferentes.

El proceso de obtención de un órgano a partir de un donante vivo repercute en múltiples aspectos, no sólo médico-quirúrgicos, sino también emocionales, sociales y económicos, tanto antes como después del trasplante (16-18). El uso de riñones procedentes de donante vivo comporta una gran responsabilidad en la evaluación y selección del donante (19-20).

La donación renal de vivo es un procedimiento relativamente seguro con unas bajas tasas de mortalidad y morbilidad que en la mayoría de las series y registros, en centros con gran experiencia en donación de vivo, han sido estimadas en un $0.03 \%$ y un $8 \%$ repectivamente (21-23). A pesar de ello, el trasplante de vivo ha estado siempre cuestionado como alternativa terapéutica precisamente por ese riesgo de morbididad y mortalidad que la nefrectomía del donante puede conllevar, sobre todo teniendo en cuenta que es la única situación en la que un sujeto sano, el donante, es sometido a una acción médica no en su propio beneficio sino en beneficio de un segundo, el receptor.

Si consideramos el riesgo al que se expone el donante, es imprescindible valorar de forma previa y meticulosa la repercusión del acto quirúrgico en ese donante potencial (24-25) y valorar el potencial beneficio que reporta el trasplante renal de donante vivo en contraposición a las desventajas, si las hubiere.

\section{Justificación del Trasplante Renal de Donante Vivo}

Entre los aspectos que justificarían la realización del trasplante renal de donante vivo podríamos destacar, la obtención de mejores resultados a corto y largo plazo en cuanto a la supervivencia de injerto y receptor, menor incidencia de necrosis tubular aguda, menor incidencia de rechazo agudo, acorta los tiempos de espera y por tanto menor tiempo en diálisis, reducción de terapia inmunosupresora, y permite la elección del mejor momento para el trasplante. A demás de suponer una ganancia emocional para el donante. Por todas estas ventajas, las guidelines europeas recomiendan informar a cerca de esta opción de trasplante en todos aquellos pacientes que puedan entrar en listas de espera (26). 


\section{Función del Injerto Rechazo Agudo}

Existe evidencia de que el retraso en la función del injerto $(27,28)$ y el rechazo agudo $(29)$ influyen de forma negativa, no sólo en la supervivencia del injerto a corto plazo, sino que también afecta en la supervivencia a largo plazo. o bviamente el riesgo de este retraso en la función es considerablemente menor en los trasplantes de donante vivo que en los procedentes de cadáver. Incluso, la incidencia de rechazo agudo es menor, y no sólo en aquellos trasplantes en los que el donante vivo está emparentado con el receptor y presentan una buena compatibilidad del HLA, sino que también es menor en aquellos trasplantes de vivo no emparentado. Este hecho es probablemente debido a que la muerte cerebral pone en funcionamiento una serie de sucesos inflamatorios no específicos que aumentarían la intensidad de la respuesta inmunológica del receptor en la fase aguda después del trasplante (30). Esta teoría está avalada por estudios en ratas en los que se demostró que la supervivencia a largo plazo de los trasplantes de riñón procedentes de donantes cadáver fue significativamente menor al compararlos con los procedentes de ratas vivas. Y además, la proteinuria y las lesiones histológicas fueron más severas en aquellas ratas a las que se les trasplantó un órgano procedente de cadáver (31).

La menor incidencia de rechazo viene determinada por dos factores: el mayor grado de identidad entre los antígenos del donante y del receptor y la menor incidencia de necrosis tubular aguda. La concomitancia de antígenos del sistema mayor de histocompatibilidad en el donante y en el receptor tiene un efecto beneficioso ya que disminuye el reconocimiento directo de las células T, así como el indirecto al reducir el número de péptidos polimórficos del donante (32). Sin embargo, este hecho es cuestionado por un estudio noruego en el que las identidades HLA A, B y DR no modificaban la supervivencia del injerto (33).

Por otra parte, el menor tiempo de isquemia fría reduce la lesión de isquemia-reperfusión, lo que disminuye el daño al injerto mediado por citoquinas asociadas al proceso inflamatorio inespecífico que se desarrolla. Al mismo tiempo la disminución de la lesión isquemia-reperfusión disminuye la susceptibilidad a respuestas aloinmunes específicas, que a través de la activación de células T mediarían en el rechazo. Esta circunstancia explica que los injertos procedentes de donante no relacionado con tiempos cortos de isquemia fría tengan mejor supervivencia que los de cadáver, con igualdad de identidades (34). A su vez, la reducción del tiempo de isquemia fría a algunos minutos reduce la necesidad de diálisis en el post-trasplante inmediato a menos del $1 \%(35)$.

El bajo riesgo de retraso en la función del injerto y de rechazo agudo, asociado a la excelente función a largo plazo de los riñones procedentes de donante vivo, confieren una gran ventaja sobre los trasplantes con riñones procedentes de donante cadáver. Revisando los registros de la UNOS (United $\mathrm{N}$ etwork for O rgan Sharing), Cecka demostró que las tasas de supervivencia del injerto a los 10 años, en aquellos trasplantes realizados en el período comprendido entre 1988 a 2000, estaban comprendidas en un $53-57 \%$ para aquellos procedentes de donante vivo contra un $38 \%$ de los procedentes de cadáver (36). Por otro lado, Ponticelli y cols informan de cifras de hasta el $73 \%$ de supervivencia del injerto a los 10 años, y además una vida media del injerto de 31.9 años en el caso de los de donante vivo contra los 18.7 años de los trasplantes de cadáver (37).

Entre otros de los inconvenientes de la utilización de órganos procedentes de cadáver, también encontraríamos el hecho de que la mayoría de donantes que mueren por patología cerebrovascular suelen estar afectos de una ateroesclerosis generalizada la cual puede alterar el funcionalismo de ambos riñones, además algunos donantes pueden ser portadores de infecciones virales latentes, o bien puede ser difícil valorar de forma fiable la función renal del donante por estar en unidades de cuidados intensivos. Por todo ello, cuando se compara el trasplante de vivo con el de cadáver se aprecia una reducción en la incidencia de retraso en la función del injerto y de rechazo agudo, permitiendo mayor supervivencia del injerto minimizando el riesgo de transmitir infecciones y tumores.

Los nuevos fármacos inmunosupresores, tacrolymus, micophenolato-mophetil, rapamycina y los anticuerpos monoclonales anti cadena alfa del receptor de la interleukina 2 (Basoliximab y Dazcizumab) han disminuido la incidencia del rechazo agudo. La menor incidencia de rechazo, permitiría una reducción de la medicación inmunosupresora con lo cual se evitarían los efectos secundarios de la misma y las consecuencias del uso crónico de fármacos esteroideos y derivados. 


\section{Supervivencia del Injerto: Donante Ideal}

El trasplante ideal sería aquel que se lleva a cabo entre hermanos gemelos idénticos, ya que no requeriría tratamiento inmunosupresor y los únicos riesgos serían los relacionados con la cirugía y la recidiva en el injerto de la enfermedad de base (38). Los registros de la UNOS informan de una supervivencia del injerto a los 5 años del $85 \%$ y una vida media del injerto de 32 años (36), siendo mejores que aquellos obtenidos con trasplantes procedentes de cadáver que mostraban una supervivencia del injerto del $62 \%$ y una vida media de 9 años (39).

En un número limitado de casos se ha encontrado que el donante es un grupo ABO incompatible con el del receptor, que hasta hace poco era considerado como una contraindicación formal para el trasplante, siendo muy elevado el riesgo de rechazo agudo, sin embargo Shimmura y cols (40) encuentran que el resultado de 67 trasplantes de donantes vivo, $A B O$ incompatibles, fue significativamente menor que los encontrados en los ABO compatibles a los 3 años del trasplante, pero no se apreciaron diferencias estadísticamente significativas cuando se evaluaron los resultados a los 4 y 8 años. El trasplante a partir de donantes $A B O$ incompatibles es todavía considerado como una opción experimental, pero los resultados de Shimmura ya han sido obtenidos en otros centros (41). En definitiva, si estuvieran disponibles varios donantes, el mejor candidato sería un gemelo idéntico 0 un hermano HLA idéntico.

\section{Momento para el Trasplante. Tiempo de diálisis}

Una importante ventaja propia del trasplante renal de donante vivo reside en el hecho de ser un procedimiento que se lleva a cabo de forma programada, electiva, lo cual permite que tanto el donante como el receptor se encuentren en las condiciones más favorables, así como la disminución del tiempo en listas de espera y a su vez en tratamiento sustitutivo con diálisis.

Diferentes estudios destacan la importancia del tiempo en diálisis como factor pronóstico del éxito del transplante renal, de forma que a mayor tiempo en diálisis, peores resultados del mismo. Montagnino y cols., observaron que en aquellos pacientes que habían recidibido diálisis durante más de 5 años la supervivencia del injerto a los 10 años fue del $45 \%$, mientras que en aquellos que habían estado menos de 5 años dicha supervivencia era del $70 \%$ (42). Cosio y cols (43) encontraron que la tasa de mortalidad post-trasplante fue del $7 \%$ en el grupo de pacientes trasplantados sin haber recibido tratamiento con diálisis previa, del 23\% en aquellos que habían estado dializados menos de 3 años y del $44 \%$ en aquellos que se habían dializado durante más de 3 años. Mange y cols (44) informaron de que el riesgo relativo de fallo del injerto también estaba relacionado con el tiempo en diálisis, siendo del 0.48 al año y del 0.18 a los 2 años en aquellos pacientes que habían no recibido diálisis previa, siendo 1 el riesgo relativo para pacientes que estuvieron sometidos regularmente a diálisis antes del trasplante. $M$ eier-Kriesch y cols (45) informaron de que el tiempo en lista de espera de 6 a 12 meses resultaba en un aumento en las tasas de mortalidad de un $37 \%$ en comparación con los que habían sido trasplantados sin diálisis previa ( preemptive transplantation ) y del $68 \%$ en aquellos que habían estado en lista de espera más de 24 meses.

\section{CONCLUSIÓN}

Estos datos remarcan que el incremento del tiempo en diálisis previo al trasplante renal está asociado con una disminución en la supervivencia del injerto y del paciente después del trasplante. Por tanto, los esfuerzos deberían encaminarse a la realización del trasplante tan pronto como fuera posible, lo cual sería más fácil llevar a cabo si hubiera disponible un donante vivo. Es por ello que el trasplante renal de donante vivo adquiere especial importancia en la infancia, ya que reduce el tiempo en lista de espera y diálisis en un momento que el crecimiento del niño exige una función renal normal. Y además permitiría elegir el momento óptimo para el trasplante en el receptor.

\section{BIBUOGRAFIA:}

1. NICHOLSON, M.L.: BMJ., 318: 409, 1999.

2. DE FELIPE, Q.; OPPENHEIMER, F.; PLAZA, J.J.: "Trasplante renal de vivo: una opción terapéutica real". Nefrología, XX: 8, 2000.

3. WOLF, J.S.; SERVINO, E.M.; NATHAN, H.N.: "National strategy to develop public acceptance of organ and tissue donation”. Transplant Proc., 29: 1477, 1997. 
4. PARJK, K.; SOO, II.K.; YU-SEUM, K. y cols.: "Results of kidney transplantation from 1979 to 1997 at Yonsei University". En Cecka, J. M.; Terasaki, P .1. ( ed ). Clinical Transplant. Los Angeles: UCLA Tissue Typing Lab. p.149-156, 1997.

5. ALBRETCHTSEN, D.; LEIVESTAD, T.; FAUCHALD, R. y cols.: "Results of the national kidney transplantation program in Norway". En: Cecka, J.M. y Terasaki, P.I. (ed.). Clinical Transplant. Los Angeles: UCLA Tissue Typing Lab. p. 207-213, 1992.

6. BEASLEY, C.L.; HULL, A.R.; ROSENTHAL, J.T.: "Living kidney donation: a survey of professional attitudes and practices". Am. J. Kid Dis., 30: 549, 1997.

7. Renal data system. USRDS 1998 annual data report. Bethesda, Md: National institute of diabetes and digestive kidney diseases, April 39, 1998.

8. BIA, M.J. y cols.: Transplantation, 60: 322, 1995.

9. SHAFFER, D.; y cols.: Arch. Surg., 4: 426, 1998.

10. PLAZA,J.J.: "Trasplante renal de vivo". Nefrología, vol. XXI: 97, 2000.

11. SANTIAGO DELPIN, E.A.; DURO GARCIA, V.: Trasplant Proc,, 31 : 2937, 1999.

12. SANTIAGO-DELPIN, E.A.; SIMMONS, R.L.; SIMMONS, R.G. y cols.: Transplant Proc., 30: 2867, 1999.

13. WOLFF, J.S.; SERVINO ,E.M.; NATHAN, H.N.: "National strategy to develop public acceptance of organ and tissue donation". Transplant Proc., 29: 1477, 1997.

14. 1MURRAY, J.E.; HARRISON, J.H.: "Surgical management of 50 patients with transplants". Am. J. Surg., 105: 205, 1963.

15. LIU, E.H.; D'ALESSANDRO, D.A.; HARDY ,M.A.: "Ethical issues in living renal donation". Transplant Proc., 35: 1174, 2003.

16. Authors for the live Organ Donor Consensus Group: JAMA, 284: 22, 2000.

17. KAULIKOVA, M.: Transplantation, 73: 1701, 2002.

18. GERALDINE, C.: Ann. Surg., 236: 120, 2002.

19. SANTIAGO-DELPIN, E.A.: Trasplantation, 64: 1374, 1997.

20. BUSTZA,C.; BRAUN, W.E.; NOVICK, A.C. y cols.: FALTA TITULO Dial Transplant, 11: 296, 1982.

21. JONHSON, E.M.; REMUCAL, M.J.; GILLINGHAM, K.J. y cols.: Transplantation, 64: 1124, 1997.

22. JONES, J.; PAYNE, W.D.; MATAS, A.J.: Tansplant Rev., 7: 115, 1993.

23. UEHLING, D.T.; MALED, G.H.; WEAR, J.B.: Urol., 76: 741, 1974.

24. WEINSTEIN, S.H.; NAVARRE, R.J.; LOENING, S.A. y cols.: J. Urol., 124: 321, 1980.

25. YASAMURA, T.; NAKAI, I.; OKA, T. y cols.: Jpn. J. Surg., 18: 252, 1988.

26. BERTHOUX, F.; ABRAMOVIC, D.; BRADLEY, B. y cols.: "European best practice guidelines for renal transplantation". Nephrol Dial Transplant, 15: 81, 2000.

27. COSIO, F.G.; PELLETIER, R.P.; FALKENHAIN, M.E. y cols.: "Impact of acute rejection and early allograftfunction on renal allograft survival". Transplantation, 63: 1611, 1997.
28. PONTICELLI, C.; VILLA, M.; CESANA, B. y cols.: "Risk factors for late kidney allograft failure". Kidney Int., 62: 1848, 2002.

29. HARIHARAN, S.; JOHNSON, C.P.; BRESNAHAM, B.A. y cols.: "Improved graft survival renal transplantation in the United States". N. Eng. J.Med., 342: 605, 2000.

30. TAKADA, M.; NADEAU, K.C.; HANCOCK, W.W. y cols.: "Effects of explosive brain death on cytokine activation of peripheral organs in rats". Transplantation, 65: 1533, 1998.

31. PRATSCHKE, J.; WILHEM, M.J.; LASKOWSKI, I. y cols.: "Influence of donor brain on chronic rejection of renal transplants in rats". J. Am. Soc. Nephrol., 12: 2474, 2001

32. FABRE, J.: "The role of polymorphic donor peptides in allograft recognition and rejection". Inmunol. Rev., 154: 21,1996

33. FOSS, A.; LEIVESTAD, T.; BREKKE, I.B.: "Unrelated living donors in 141 kidney transplantations". Transplantation, 66: 49, 1998.

34. FANGMAN, J.; FRÜHAUF, N.; OLDHAFER, K.: "Living related and cadaveric kidney transplantation. What are de major differences?". Transplant Proc., 31: 203, 1999.

35. STREEM, S.B.; NOVICK, A.C.; STEINMÜLLER, A.R. y cols.: "Flank donor nephrectomy: efficacy in the donor and the recipient". J. Urol., 141: 1099, 1989.

36. CECKA, J.M.: "The UNOS renal transplant registry". In: Cecka JM and Terasaki P. Eds. Clinical Transplants 2000. Los Angeles, UCLA 2001.

37. PONTICELLI, C.: "Altruistic living renal transplantation". J. Nephrol., 16: 86, 2003.

38. TILNEY, N.L.: "Tansplantation between identical twins: a review". World J. Surg., 10: 381, 1986.

39. GJERTSON, D.W.; CECKA, J.M.: "Living unrelated kidney transplantation". Kidney Int 58: 491, 2000.

40. SHIMMURA, H.; TANABE, K.; ISHIKAWA, N. y cols.: "Removal of antiA/B antibodies with plasmapheresis in ABO-incompatible kidney transplantation". Ther. Apher., 4: 395, 2000.

41. RYDBERG, L.: "ABO-incompatibility in solid organ transplantation". Transfus. Med., 11: 325, 2001.

42. MONTAGNinO, G.; TARANTINO, A.; CESANA, B., y cols.: "Prognostic factors of longterm allograft survival in 632 Cya-treated recipients of a primary renal transplant". Transpl. Int., 10: 268, 1997.

43. COSIO, F.G.; AlLAMIR, A.; YIM, S. y cols.: "Patient survival after renal transplantation: the impact of dialysis pre-transplant". Kidney Int., 53: 762, 1998.

44. MANGE, K.C.; JOFFE, M.M.; FELDMAN, H.I.: "Effect of the use or non-use of long-term dialysis on the subsequent survival of renal transplantation from living donors". N. Eng. J. Med., 344: 726, 2001.

45. MEIER-KRIESCHE, H.U.; PORT, F.K.; OJO, A.O. y cols.: "Effect of waiting time on renal transplant outcome". Kidney Int., 58: 1311, 2000. 